\title{
Rotational Evolution of Self-Gravitating Aggregates with Cores of Variable Strength
}

\author{
Paul Sánchez ${ }^{\mathrm{a}, *}$, Daniel J. Scheeres ${ }^{\mathrm{b}}$ \\ ${ }^{a}$ Colorado Center for Astrodynamics Research, University of Colorado Boulder, Boulder, Colorado, 431 UCB, 80309-431, USA \\ ${ }^{b}$ Department of Aerospace Engineering Sciences, University of Colorado Boulder, Boulder, Colorado, 429 UCB, 80309, USA.
}

\begin{abstract}
This paper presents a study, through the use of a Soft-Sphere Discrete Element method, of possible deformation and disruption patterns of spinning, self-gravitating spherical aggregates with cores of variable strengths. We present this study as a complement to our previous study about aggregates with strong cores that also provided some insight into the occurrence of surface shedding. It is observed that the inclusion of a weak core produces a very symmetric deformation pattern and even a shape that resembles that of asteroid 25143 Itokawa though this only happens for the weakest of the tested cores. At this level of shell strength, and in agreement with our previous studies, most aggregates fission off coherent pieces of the original bodies, which could potentially form binary systems. Additionally, we try to understand how strong, relative to the shell, the core has to be to prevent it from failing before the shell does.
\end{abstract}

Keywords: Asteroids, Rotation, Granular, Interior, Itokawa, Simulation

\section{Introduction}

During the last two decades, the study of asteroids as self-gravitating granular systems has gained importance. Much of this interest and inter-disciplinary approach to their study has been influenced by different space missions to asteroids and comets. In particular, the images and samples obtained by the JAXA mission Hayabusa to asteroid Itokawa (Fujiwara et al., 2006; Yano et al., 2006; Miyamoto et al., 2007) supported the idea that small asteroids are self-gravitating aggregates that are held together by not only gravitational, but also, cohesive and adhesive forces (Jewitt et al., 2013, 2014; Hirabayashi et al., 2014; Rozitis et al., 2014; Hirabayashi and Scheeres, 2015). Furthermore, these images showed surfaces that were far from homogeneous in structure and that, much more interestingly, presented size-segregated particles.

If the surface of asteroids present this heterogeneity, it seems only logical that their interiors could also be heterogeneous. Prompted by the discovery of active asteroids P/2013 R3 and P/2013 P5 (Jewitt et al., 2013, 2014),

${ }^{*}$ Corresponding author

Email addresses: diego.sanchez-lana@colorado.edu (Paul Sánchez), scheeres@colorado.edu (Daniel J. Scheeres) 
Hirabayashi et al. (2015) analyzed the disruption mechanism of self-gravitating aggregates with a strong core and a weak shell. This research concluded that this morphology produces surface shedding in a rotating self-gravitating aggregate with some degree of cohesive strength. Additionally, it has also been suggested by Tardivel et al. (2018) that the presence of a strong core, together with kinetic sieving in the surface of asteroids 2008 EV5 (Busch et al., 2011) and 2000 DP107 (Naidu et al., 2015), could have produced the cavity on their equators through rotational fission.

Of course, there is no reason to believe that a strong core is the only type of heterogeneity that could exist or that heterogeneities have a single origin. Heterogeneities could appear as a result of the inclusion of grains of different materials which would produce different material and bulk densities, different cohesive and adhesive forces and different packing fractions that would in turn have an effect on the overall strength of certain regions of the asteroid. Also, as the strength of the grains that form an asteroid are linked to the different crystallographic phases of the materials that form them, grains of different materials would produce grains with different morphologies; more or less angular edges or irregular shapes will have an effect in the geometric interlocking of particles which will in turn have an effect on the angle of friction of the system. Additionally, the strength of the grains themselves will likely determine their size distribution, regardless of the production method (impacts or pulverization due to thermal cycling). In this paper we first study the dynamics of cohesive, self-gravitating aggregates with a weak core where the source of the strength is the cohesive forces between the particles. With this objective in mind, we follow a very similar approach to Hirabayashi et al. (2015) and after that we choose two core sizes to observe, step by step, the effect of the variation of the strength of the core. We do this so that a coherent picture, if still very basic, about the role of size and strength of interior heterogeneities can be understood.

This paper is organized as follows: first we will describe the physical model and the simulation method that is going to be used for this work. Then we will describe the geometry of the aggregates and how we varied it. After this, we will describe the initial deformation and disruption patterns of aggregates with a constant core strength, but variable core size. At this point we will focus on a couple of geometries to observe the effect of a variable core strength and constant core size. Finally we will relate our results to asteroids 1999 KW4 and 25143 Itokawa to then establish conclusions about their internal structure and superficial morphology and how they are related to each other.

\section{Internal Core Model}

Figure 1 shows the model we are going to be using for this study. We suppose that our test body is spherical, that it is uniformly rotating around the $z$ axis and that it is granular in nature so that it can be deformed or disrupted at high enough spin rates. The body itself is not homogeneous, but it has a concentric spherical core that is weaker than its outer shell. We assume that this difference is originated only due to different tensile strengths whereas all other characteristics (bulk density, porosity, angle of friction) are left unchanged. This structure guarantees that by the time 
the shell starts to fail, the core will not present any resistance; furthermore, it should prompt the failure of the shell at a lower spin rate than had the aggregate been homogeneous.

The bulk density, the total radius and the gravitational constant are denoted as $\rho, R$ and $G$, respectively. We normalize lengths, body forces, spin rates and stress tensors by $R, \pi \rho G, \sqrt{\pi \rho G}$ and $\pi \rho^{2} G R^{2}$, respectively. With this normalization, the sphere radius is denoted as 1 and the radius of the internal core is defined as $R_{b}$, so the thickness of the surface shell is given as $1-R_{b}$.

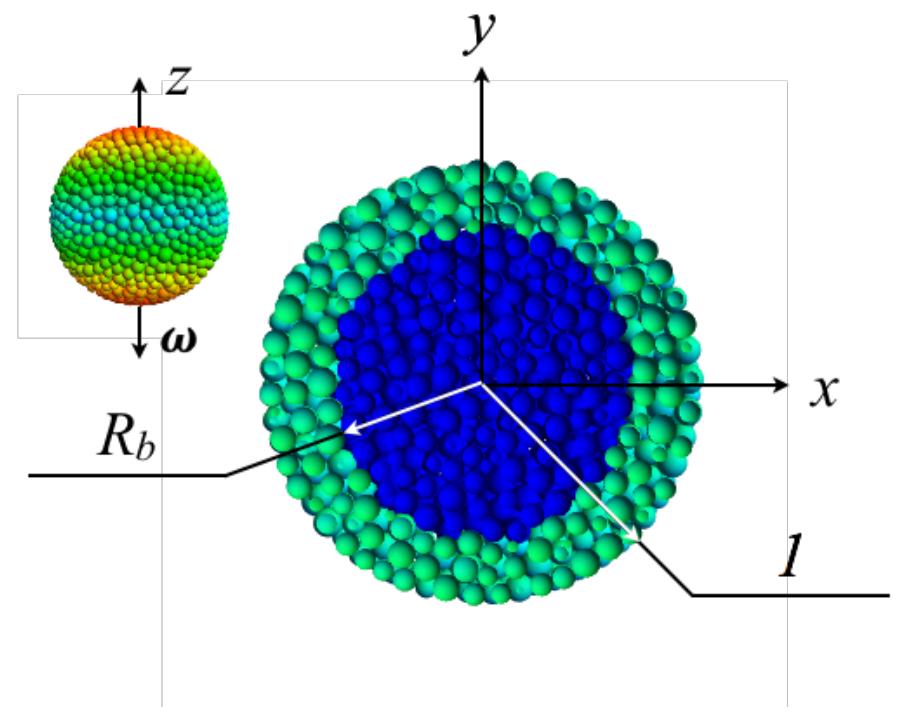

Figure 1: Cross-sectional cut of an aggregate with the internal core $\left(R_{b}=0.7\right)$. The sphere is assumed to be spinning constantly along the $\mathrm{z}$ axis. The normalized radii of the sphere and the internal core are given as 1 and $R_{b}$, respectively. The sphere on the top left corner shows the initial configuration of the aggregates and it is rotated $90^{\circ}$ with respect to the cross-sectional cut. The colours represent latitude.

\section{Simulation Method}

The simulation program that is used for this research applies a Soft-Sphere Discrete Element Method (SSDEM) to simulate a self-gravitating granular aggregate (Cundall, 1971; Cundall and Hart, 1992; Sánchez and Scheeres, 2011). The particles, modeled as spheres that follow a predetermined size distribution, interact through a soft-repulsive potential when in contact. This method considers that two particles are in contact when they overlap. When this happens, normal and tangential contact forces are calculated (Herrmann and Luding, 1998). The former is modeled by a hertzian spring-dashpot system and is always repulsive, keeping the particles apart; the latter is modeled with a linear spring that satisfies the local Coulomb yield criterion. The normal elastic force is modeled as

$$
\overrightarrow{\mathbf{f}}_{e}=k_{n} \xi^{3 / 2} \hat{\mathbf{n}},
$$


the damping force as:

$$
\overrightarrow{\mathbf{f}}_{d}=-\gamma_{n} \dot{\xi} \hat{\mathbf{n}}
$$

and the cohesive force between the particles is calculated as

$$
\overrightarrow{\mathbf{f}}_{c}=-2 \pi \frac{r_{1}^{2} r_{2}^{2}}{r_{1}^{2}+r_{2}^{2}} \sigma_{y y} \hat{\mathbf{n}}
$$

where $r_{1}$ and $r_{2}$ are the radii of the two particles in contact and $\sigma_{y y}$ is the tensile strength of this contact, which is given by a cohesive matrix formed by the (non-simulated) interstitial regolith (Sánchez and Scheeres, 2014). Then, the total normal force is calculated as $\overrightarrow{\mathbf{f}}_{n}=\overrightarrow{\mathbf{f}}_{e}+\overrightarrow{\mathbf{f}}_{c}+\overrightarrow{\mathbf{f}}_{d}$. In these equations, $k_{n}$ is the elastic constant, $\xi$ is the overlap of the particles (in length units), $\gamma_{n}$ is the damping constant (related to the dashpot), $\dot{\xi}$ is the rate of deformation and $\hat{\mathbf{n}}$ is the unit vector joining the centres of the colliding particles. This dashpot models the energy dissipation that occurs during a real collision.

The tangential component of the contact force models surface friction statically and dynamically. This is calculated by placing a linear spring attached to both particles at the contact point at the beginning of the collision (Herrmann and Luding, 1998; Silbert et al., 2001) and by producing a restoring frictional force $\overrightarrow{\mathbf{f}}_{t}$. The magnitude of the elongation of this tangential spring is truncated in order to satisfy the local Coulomb yield criterion $\left|\overrightarrow{\mathbf{f}}_{t}\right| \leq \mu\left|\overrightarrow{\mathbf{f}}_{n}\right|$.

Rolling friction (Ai et al., 2011; Sánchez and Scheeres, 2016) has also been implemented in order to mimic the behaviour of aggregates formed by non-spherical grains. Particles are subjected to a torque that opposes the relative rotation of any two particles in contact. This model "places" a winding spring of sorts that is extended when two contacting particles roll on one another along with a velocity dependent dashpot. Much like the tangential spring that models static friction between particles in our code, this spring also breaks and allows rotation when a certain limit has been reached. This torque, similar to surface-surface friction, is implemented as linearly dependent on the relative angular displacement of any two particles in contact and has a limiting value of:

$$
M_{r}^{m}=\mu_{r} R_{r}\left|\overrightarrow{\mathbf{f}}_{n}\right|
$$

where $\mu_{r}$ is the coefficient of rolling resistance and $R_{r}=r_{1} r_{2} /\left(r_{1}+r_{2}\right)$ is the rolling radius.

The viscous damping torque $M_{r}^{d}$ is assumed to be dependent on the relative rolling angular velocity $\dot{\theta}_{r}$ between the two particles in contact and the damping constant $C_{r}$ :

$$
M_{r, t+\Delta t}^{d}= \begin{cases}-C_{r} \dot{\theta}_{r} & \text { if }\left|M_{r, t+\Delta t}^{k}\right|<M_{r}^{m} \\ -f C_{r} \dot{\theta}_{r} & \text { if }\left|M_{r, t+\Delta t}^{k}\right|=M_{r}^{m}\end{cases}
$$

This last equation contains a term $f$, which determines whether the viscous damping torque is only active before the contact rolling torque is fully mobilised $(f=0)$ or if it is always present $(f=1)$. For simplicity we have chosen $f=0$ for our simulations. 
This implementation allows our simulations to reach angles of friction of up to $\approx 35^{\circ}$ as evaluated by the DrukerPrager yield criterion (Sánchez and Scheeres, 2012). This value for the angle of friction is typical of geological aggregates, though friction angles of $\sim 40^{\circ}$ are not rare. This implementation of rolling friction is similar to that of surface friction, but depends on the incremental relative rotation, as such we have effectively implemented twisting friction as well (Sánchez and Scheeres, 2016).

The normalized parameters of the model are as follows: $k_{n}=6.1 \times 10^{-7}, k_{t}=2.1 \times 10^{-6}, \gamma_{n}=67, \mu=0.5$, and $\mu_{r}=0.8$

\section{Geometry of the Aggregates}

To obtain the initial configuration of the aggregates, we leave the particles (initially cohesionless and frictionless) to coalesce only under the influence of their mutual gravitational interactions. Then, the particles are encapsulated inside a perfectly solid sphere that shrinks to a predetermined size. This size has been pre-determined from previous simulations so that the particles are forced into a close-to-perfect spherical shape. For all simulations, the initial filling fraction is $\approx 0.63$. When the set-up procedure is finished, friction and cohesive forces are applied (producing an angle of friction of $\approx 35^{\circ}$ ) and the simulation is ready to start. Once the simulation starts, the aggregate is spun up in small, discrete increments of a normalized spin rate of $5.5 \times 10^{-3}$ every $3000 \mathrm{~s}$ around the centre of mass and the disruption process is observed. This time step was long enough to allow the aggregates to safely reshape before the next spin-up event. This increment in angular velocity was added to the entire body as well as to each individual particle so that failure is not artificially triggered. The simulation has periodic boundaries and so, in general, we will not analyze the aggregates after fissioned particles go through them. The aggregates rotate in the $-\mathrm{z}$ direction to facilitate the observation of the reshaping and disruption process through the graphical interface of the simulation code.

For this research, to observe the effect of an internal core in a self-gravitating aggregate, we have chosen to have cores whose radii are $0.5,0.6,0.7,0.8$ and 0.9 . The particles forming the core are subjected to cohesive forces that are ten times as weak as those felt by the particles of the shell. From the analytical model and the simulations carried out by Hirabayashi et al. (2015), it is known that these aggregates would fail initially at the core, much more so now that the core has been severely weakened. All the aggregates used in the simulations are formed by 3000 perfectly spherical particles with normalized, randomly chosen radii between 0.025 and 0.035 . We do this to avoid the crystallization of the particles.

The tensile strength of the core is given by $\sigma_{t c}=0.1 \sigma_{t s}$, where $\sigma_{t s}$ is the chosen tensile strength between the particles of the shell, and the tensile strength between particles in the interface between the shell and the core is set to be $\sigma_{t i}=0.5 \sigma_{t s}$. The corresponding cohesive strength of these regions of the aggregates $\left(\sigma_{c c}, \sigma_{c s}, \sigma_{c i}\right)$ keep the same proportionality as $\sigma_{c}=\sigma_{t} \tan \theta$, where $\theta$ is the angle of friction. Notice also that the tensile strength between two 
particles $\left(\sigma_{y y}\right)$ is related to $\sigma_{t}$ by the expression ${ }^{1}$

$$
\sigma_{t}=\sigma_{y y} \frac{\phi \cos \left(45^{\circ}\right)}{\sqrt{3}}
$$

where $\phi$ is the filling fraction.

\section{Results}

\subsection{Initial Deformation and Failure}

To analyze the deformation of the aggregates, we use the definition of ellipticity used by Bottke et al. (1999); if the semi-major axes of the $\mathrm{DEEVE}^{2}$ of an aggregate are defined as $a_{1} \geq a_{2} \geq a_{3}$, and the axes ratios as $q_{2}=a_{2} / a_{1}$ and $q_{3}=a_{3} / a_{1}$, the ellipticity is $\epsilon=1-\frac{1}{2}\left(q_{2}+q_{3}\right)$. For a perfectly spherical aggregate, $\epsilon=0$. Given that we are effectively reducing the the overall strength of the aggregates by increasing the size of the weak core, it would have been expected to have an observable difference in the ellipticity of the deformed body, but this was not found. The inclusion of a weak core in the aggregates had one visible effect: deformation and disruption started at lower spin rates with larger cores, as is evident in Fig. 2. In this figure we have plotted not only the normalized spin rate evolution of the aggregates with weak cores versus time, but also the evolution for the homogenous ones that could be regarded as limiting cases. Notice that $\sigma_{c}$ is the normalized cohesive strength of the homegeneous aggregates.

Contrary to aggregates with strong cores, one of the main differences that can be clearly observed with weak core aggregates is that the latter $\left(R_{b} \geq 0.6\right)$ will develop a very symmetrical, ellipsoidal shape before fission takes place and the equatorial bulge will be formed by the plastic deformation of the shell as it is pushed out by the core whilst the poles push in (Hirabayashi and Scheeres, 2015). This also produces very symmetrical ellipsoidal bodies before fission and disruption processes take place. A very strong core on the other hand will produce granular flow from the midlatitudes. This will flatten the mid-latitude regions (Scheeres, 2015) and form a sharp equatorial ridge, creating little to no deformation of the core (Hirabayashi et al., 2015). Aggregates with homogeneous interiors seem to combine these two limiting behaviours (Sánchez and Scheeres, 2016) as they first have an elastic deformation, followed by a plastic deformation and finally a localized surface flow that will result in an asymmetrical shape.

As an example, Fig. 3 shows the motion of the particles and deformation of the aggregate with $R_{b}=0.7$ an instant before the onset of disruption. The blue dots represent the original position of the particles across a cross-sectional cut that passes through the centre of mass of the aggregate and is perpendicular to one of the axes of the simulation, whereas the arrows show the particles' displacement.

\footnotetext{
${ }^{1}$ In Sánchez and Scheeres (2016), $\sigma_{t}$ was denoted $\sigma_{y y}$. Also the denominator should have a square root and not a cubic root; this was a typographical error.

${ }^{2}$ DEEVE: Dynamically Equivalent Equal Volume Ellipsoid.
} 


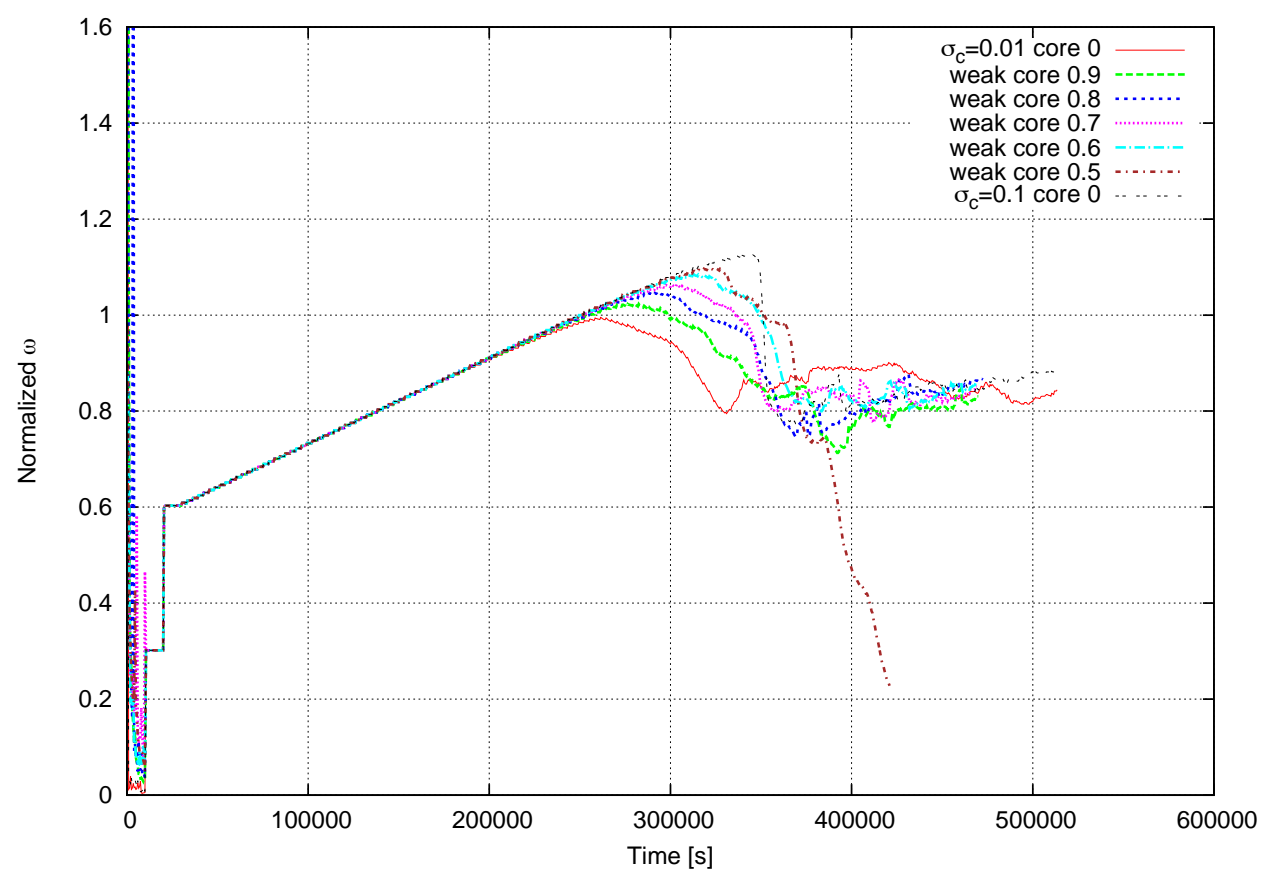

Figure 2: Normalized spin rate evolution of the simulated aggregates. The red and black lines correspond to the homogeneous aggregates (core 0). $\sigma_{c}$ is the normalized cohesive strength of the homegeneous aggregates.
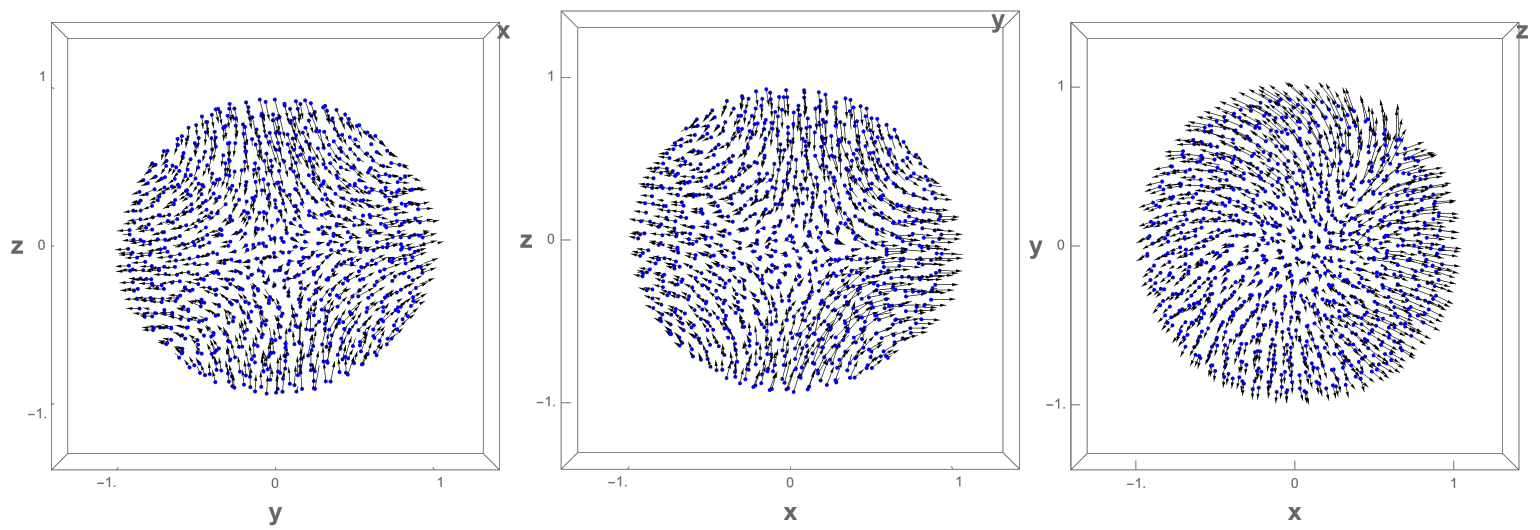

Figure 3: Deformation of an aggregate with $R_{b}=0.7$. The images show the original position of the particles across cross-sectional cuts of the aggregate (blue dots) perpendicular to the $\mathrm{x}$ (left), $\mathrm{y}$ (centre) and $\mathrm{z}$ (right) axes. The arrows show the displacement of the particles an instant before disruption. 


\subsection{Global Failure and Disruption}

Figure 4 shows the disruption patterns of the simulated aggregates whilst they occurred. From them it can be observed that starting with $R_{b}=0.9$, after an initial deformation, the disruption pattern continues the trend marked by the original homogeneous aggregate (Sánchez and Scheeres, 2016), that is, there is a particle-by-particle detachment. As the size of the core decreases, the disruption pattern changes and the aggregate starts to eject coherent pieces formed by many particles. However, for $R_{b}=0.5$ this changes again to a particle-by-particle detachment.

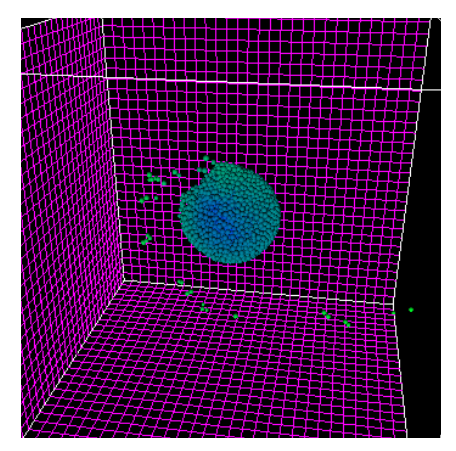

(a) $R_{b}=0.9$

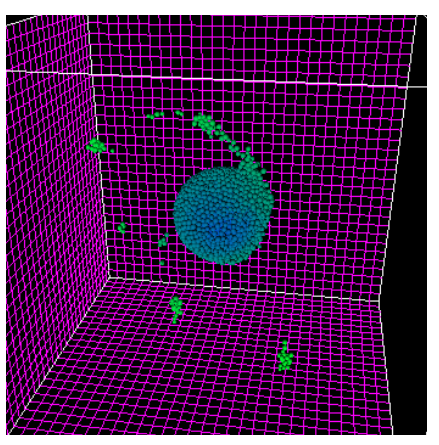

(b) $R_{b}=0.8$

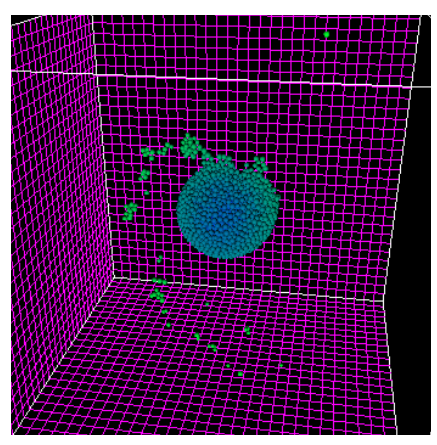

(c) $R_{b}=0.7$

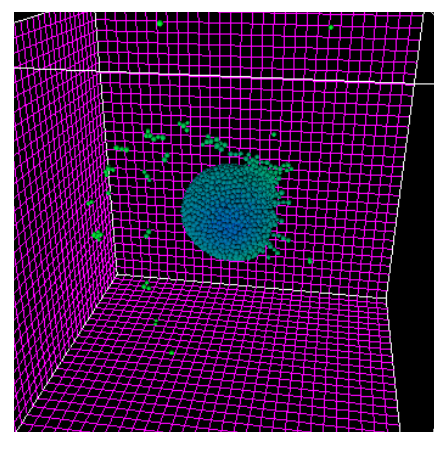

(d) $R_{b}=0.6$

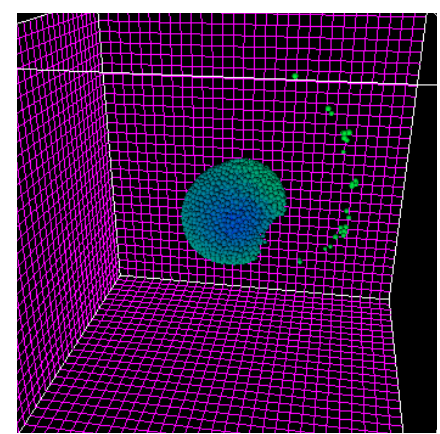

(e) $R_{b}=0.5$

Figure 4: Disruption pattern of five cohesive, self-gravitating spherical aggregates with different core sizes $\left(R_{b}\right)$. The coulours show the distance of the particles to the centre of mass of the system from blue (close) to green (far).

Though the initial deformation for all of the aggregates was symmetric towards an oblate ellipsoid shape, the final shapes after disruption were not. As pointed out by Hirabayashi et al. (2015), as homogeneous as we would like our aggregates to be, by construction they present internal weak points where the body (as any real aggregate) can fail more easily. This fact, combined with the continuous injection of angular momentum coupled with the ejection of material that it provoked at higher spin rates, produced non-symmetrical shapes. This is the reason for the local nature of the region of greater deformation. Once the aggregate deforms in one region, its internal stress is diminished as the angular velocity has to decrease to conserve angular momentum. As can be observed from Fig. 5, the core and shell show signs of asymmetric deformation for all core sizes, but it is much more prominent for $R_{b}=0.5$, where the 
shell has developed a dent and the core is much more deformed. All images represent a cross-section perpendicular to the rotation axis. Additionally, if we compare these images to the original configuration presented in Fig. 1, the

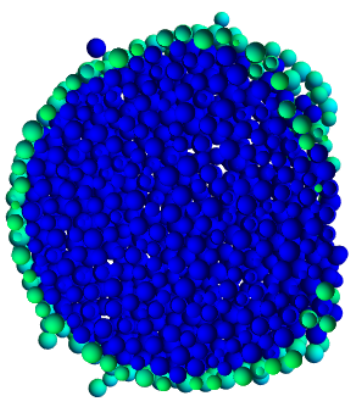

(a) $R_{b}=0.9$

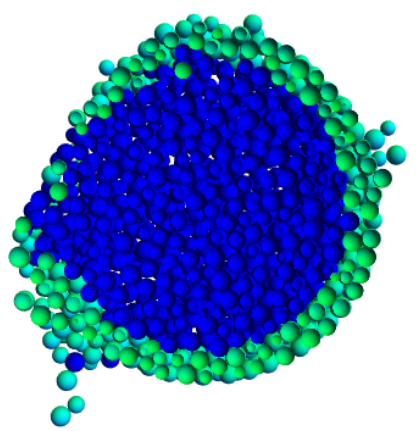

(b) $R_{b}=0.8$

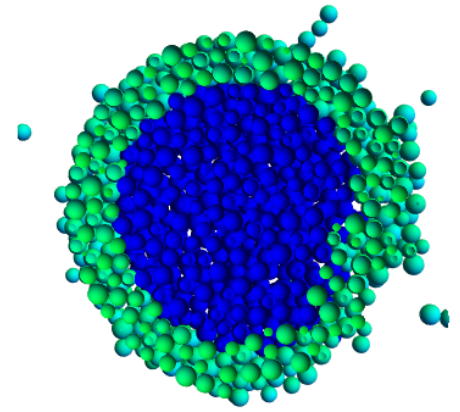

(c) $R_{b}=0.7$

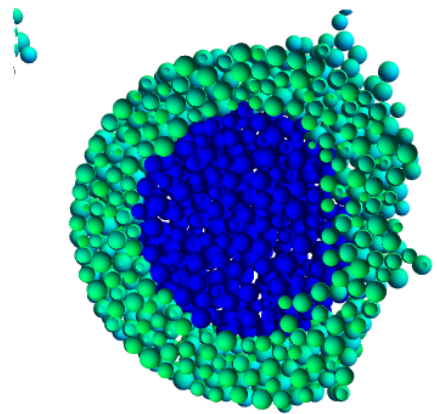

(d) $R_{b}=0.6$

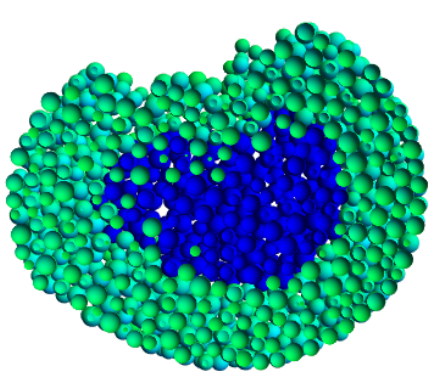

(e) $R_{b}=0.5$

Figure 5: Global deformation of the simulated aggregates after the first disruption process has taken place. The shell is coloured in green and the core in blue. All images represent a cross-section perpendicular to the rotation axis.

formation of large voids, especially in the core, is evident. This supports the findings of Sánchez and Scheeres (2012) and Hirabayashi and Scheeres (2015), where the authors found that the deformation of the aggregates' interior would lead to increased porosities as long as realistic angles of friction and particle-particle friction were considered.

All the simulations continued after this first disruption process to observe the dynamics of the systems. This showed that an increase in angular velocity meant the continuation of the disruption processes that had been initially observed, except for $R_{b}=0.5$. For this aggregate, and due to the dent that had been induced in the shell, the deformation and disruption process changed dramatically and this was one of the most interesting findings. The next section will provide a detailed account of this particular aggregate, its interior, its shell and the disruption process. 


\subsection{Making Itokawa}

The image presented in Fig. 4(e) is the initial deformation and disruption of the aggregate, but contrary to the rest of the simulations, its shape is far from being ellipsoidal and it actually has a dent in the outer shell. This simulation (as well as the rest) was continued to observe the subsequent evolution of shape and disruption and the results are shown in Fig. 6

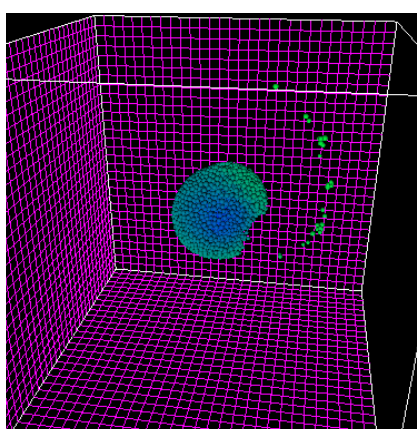

(a)

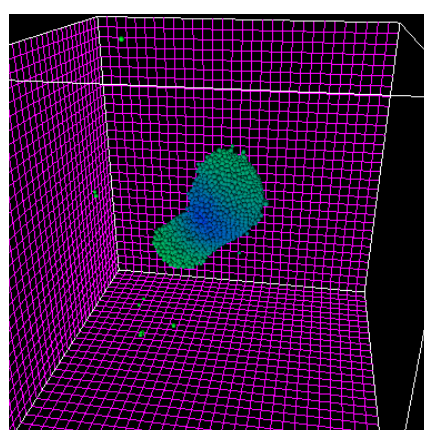

(b)

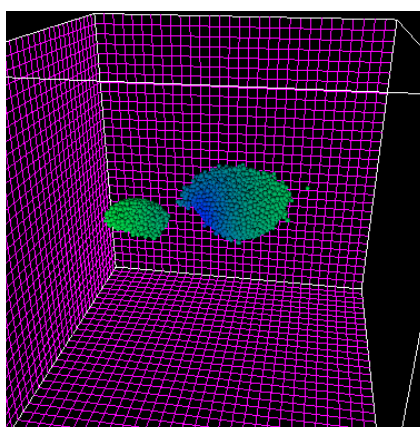

(c)

Figure 6: Further evolution of the $R_{b}=0.5$ aggregate. From left to right, (a) the initial deformation and disruption process, (b) the Itokawa-like stage and (c) complete fission of the head and body.

As it can be observed, the shape acquired by this aggregate, with this particular geometry and distribution of strength, is very similar to asteroid 25143 Itokwa, which was visited and studied by the Hayabusa mission (JAXA) (Fujiwara et al., 2006). In our simulations, this aggregate, having a very weak core, first forms what seems to be a dent in the shell, then stretches to a non-ellipsoidal shape, with a very distinctive "head" and "body", and finally breaks into two non-symmetrical pieces. Notice that this configuration is stable and it only changes when it is spun up even further. Though this outcome is welcome, we cannot say that this is representative of Itokawa as the asteroid has some very unique features that we should first at least try to explain.

Figure 7 displays a more detailed view of this aggregate, showing views from the sides((a) and (b)), top (d) and a cross-sectional cut (c) across a quasi-plane of symmetry. In this figure, the colours on the surface only show different latitudes; internally, the particles that formed the core are coloured in magenta, whereas those forming the shell are coloured in light green. From these images it is evident that the shell as well as the core are very deformed compared to their initial spherical shapes. In fact, the shell was stretched to the point that some of the core can be observed through it whilst at the same time it formed the head leaving a stretched-out core to form the interior of the body.

In Fig. 8 we have given the particles of the shell two different colours so that its stretching is more evident. This allows us to observe that it is across the top and bottom of the aggregate that the shell has stretched the most. Furthermore it is here that some of the subsurface material can be observed. More specifically, this happens from 


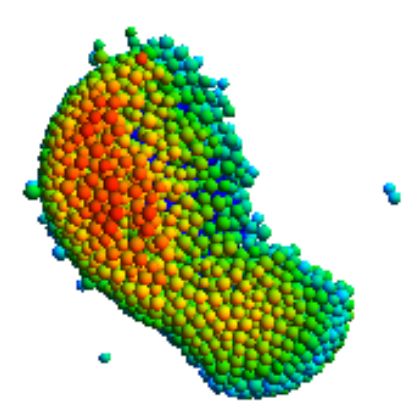

(a) Left side

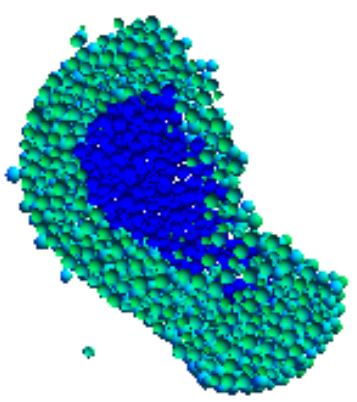

(c) Cross-section

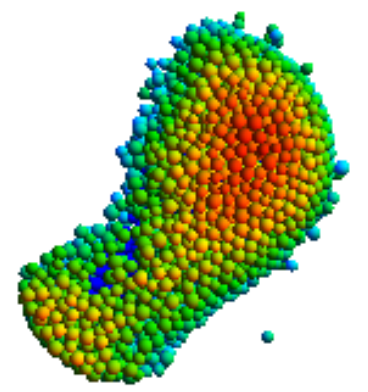

(b) Right side

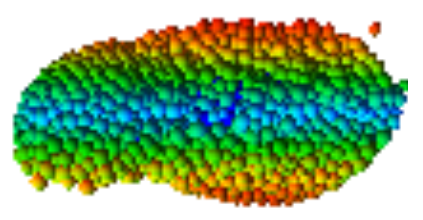

(d) Top

Figure 7: Different views of the $R_{b}=0.5$ aggregate showing how the outstretched shell forms the head upon rotation. The colours of the shell show different latitudes, whereas the colours of the interior show the shell and core regions in green and blue colours respectively.

the body towards the neck and in the back, exactly the places where asteroid Itokawa shows ponds containing small regolith and dust.

It has been previously established that asteroid Itokawa could be a "contact binary" (Scheeres, 2007). However, though we cannot disregard that formation mechanism, given the results of this research we could suggest a different formation scenario. For that, asteroid Itokawa would have had to start its shaping process (or at least the latest one) as a quasi-spherical body with a very weak core. This proto-Itokawa would have then been spun up to disruption rates due to the YORP effect (Rubincam, 2000) that would have led to the stretching of the outermost layer of material, so exposing some of the subsurface material. This picture however presents a problem as the strength of a cohesive granular material seems to be strongly related to the size distribution of its constitutive grains (Sánchez and Scheeres, 2014), which would imply that the subsurface material is actually stronger than the outermost layer; however, there is a solution for this conundrum. The segregation observed on Itokawa's surface was first attributed to the Brazil Nut effect by Asphaug et al. (2001); this problem has more recently been investigated by Sánchez et al. (2010) and Perera et al. (2016). These papers investigate the possibility of a particle size segregation process that is driven by multiple, 


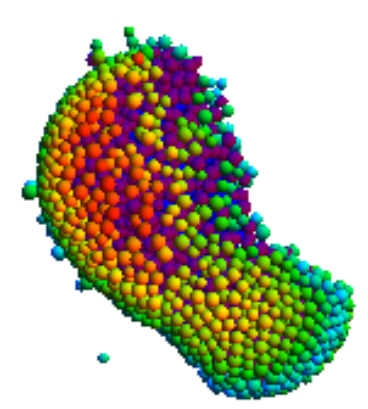

(a) Left side

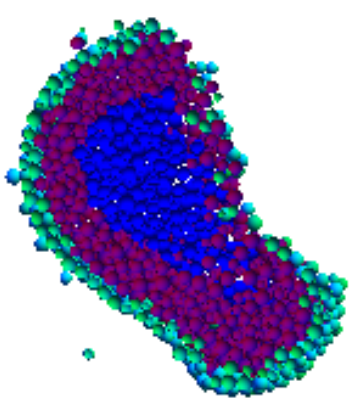

(c) Cross-section

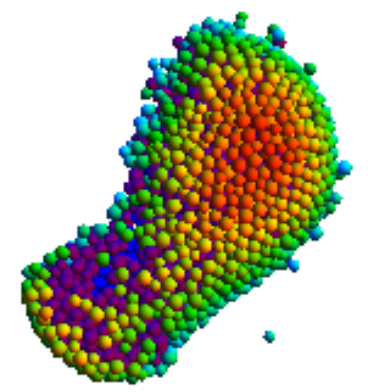

(b) Right side

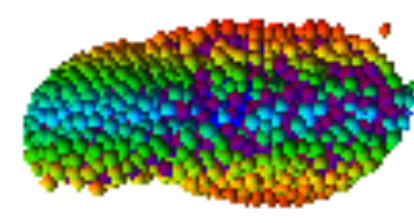

(d) Top

Figure 8: Detail of the deformation of the $R_{b}=0.5$ aggregate showing how the outstretched shell is thinnest on the top and the back sides of the aggregate.

non-catastrophic impacts on an asteroid. Both found that impacts could lead to a depletion of the smaller particles in the outermost layer of a granular asteroid though they attributed this to different mechanisms. Regardless of that, this segregation happens only on the very surface of the aggregate, leaving the interior intact. In our simulation, the entirety of the core $\left(R_{b}=0.5\right)$ and most of the shell correspond to this interior that is not affected by the segregation process. This configuration is more complex than the one we have tested here, but if the size separation takes place close to the surface, it should not cause a different behaviour. However, notice that with this we are not attempting to explain the origin of the internal structure of asteroid Itokawa, but merely the morphology of its surface. As we have shown before (Hirabayashi et al., 2015) spherical aggregates with homogeneous interiors will fail at the centre first, which means that a weak core will present even less resistance. Incidentally, if the strength of the different regions of our simulated aggregate was the result of different bulk densities, perhaps correlated with different cohesive forces between the particles, the head should be denser than the body, which is precisely what has been suggested about Itokawa (Scheeres and Gaskell, 2008; Lowry et al., 2014). 


\section{How strong is strong?}

Up to this point we have described and analyzed the results obtained by keeping the strength of the core constant and changing its size, as was done by Hirabayashi et al. (2015). However, given that in homogeneous and weak-core aggregates, the core would always fail before the shell, it is also interesting to investigate the point at which a core is strong enough to allow the shell to fail first. In order to do this we have chosen to analyze two core sizes, $R_{b}=0.5$ and $R_{b}=0.7$. The former is able to produce a shape similar to that of asteroid Itokawa, whereas the latter provides an analog for the shape of asteroid 1999 KW4 (Sánchez, 2015; Hirabayashi et al., 2015).

The procedure is as follows: for each core size, as simulations have been carried out at both ends of the strength (0.01-0.50), starting with the weakest core, we increase the strength of the core alone by 0.02 until the strength of the shell is reached. After this, the strength of the core will be increased by 0.1 until the strength of the core is five times the strength of the shell. The strength of the interface is increased from 0.05 through 0.15 in steps of 0.01 . Table 1 , summarizes the values of normalized cohesive strength that we have used in our simulations.

Table 1: Normalized cohesive strength of the shell $\left(\sigma_{c s}\right)$, interface $\left(\sigma_{c i}\right)$ and core $\left(\sigma_{c c}\right)$.

\begin{tabular}{ccc}
\hline Shell & Interface & Core \\
\hline \multicolumn{3}{c}{ Weak Core $\sigma_{c c}$} \\
\hline 0.1 & 0.05 & 0.01 \\
0.1 & 0.06 & 0.03 \\
0.1 & 0.07 & 0.05 \\
0.1 & 0.08 & 0.07 \\
0.1 & 0.095 & 0.09 \\
\hline & Strong Core $\sigma_{c c}$ \\
\hline 0.1 & 0.11 & 0.15 \\
0.1 & 0.12 & 0.20 \\
0.1 & 0.13 & 0.30 \\
0.1 & 0.14 & 0.40 \\
0.1 & 0.15 & 0.50 \\
\hline
\end{tabular}

We varied strength in the aggregates in this specific manner so that the original simulations with weak cores (previous sections), homogeneous interiors (Sánchez and Scheeres, 2016) and strong cores (Hirabayashi et al., 2015) can be taken as part of this sequence of simulations. Additionally, we hope this will allow us to provide a unified picture of the effect that the internal structure of a self-gravitating aggregate has on the evolution of its shape and 
eventual disruption. We hope the reader appreciates that there are many other factors, such as packing fraction, contact networks, density variations, shape and position of a "core" among the most important, that have not been studied yet. In spite of this, the fact that a model, as simple as the one used for all these studies can present such a wealth of behaviours that can be related to observed asteroids is remarkable and therein lies its importance.

Figure 9 shows the variation of the normalized critical spin rate in relation to the variation of the normalized cohesive strength of the core of each of the chosen aggregates. The critical spin rate is defined as the peak spin rate that an aggregate can reach before plastic deformation or failure. As shown by Sánchez and Scheeres (2012), the violation of the Druker-Prager criterion (which indicates structural failure) happened when the body was deformed and its angular velocity changed. As the normalized cohesive strength of the shell for all aggregates in this study is 0.1 , it seems that for the core to be seen as solid from the perspective of the shell, the former must be at least between 3 to 4 times as strong as the latter.

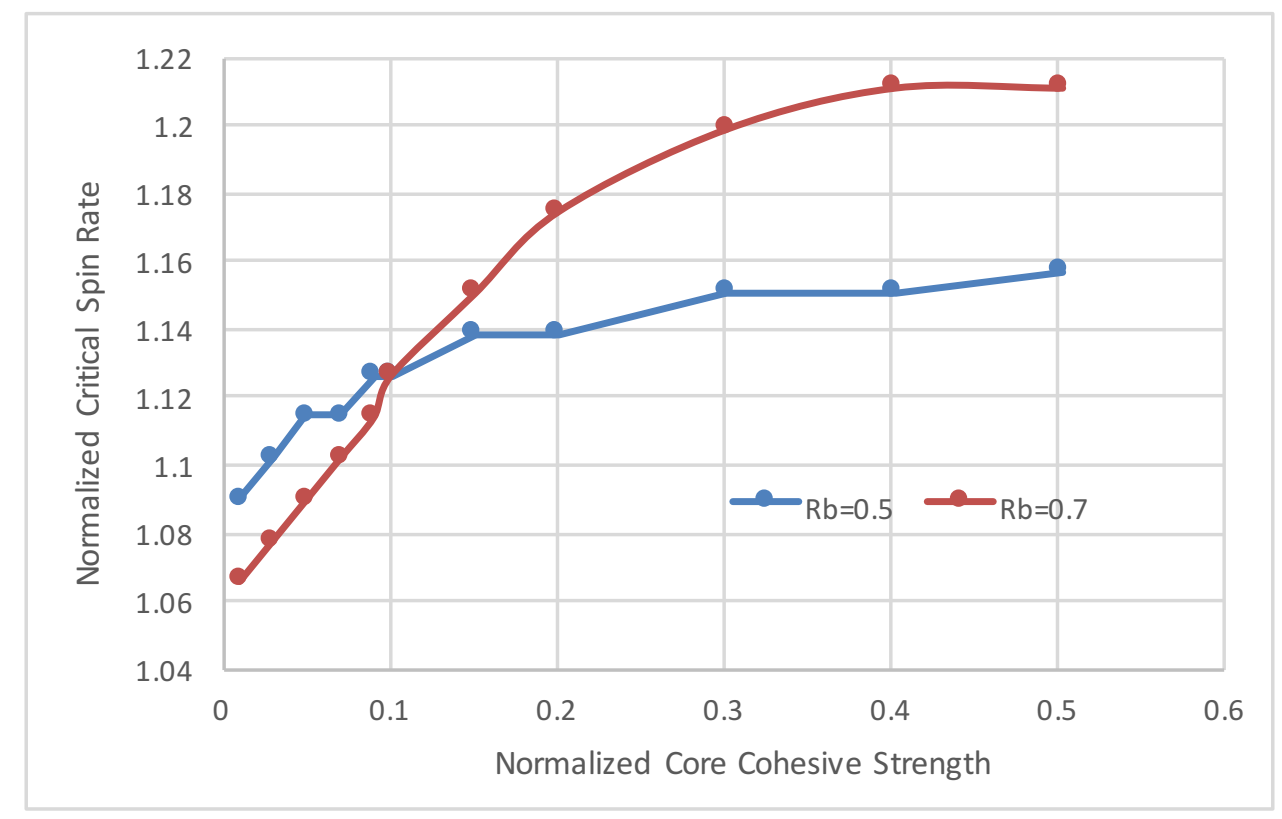

Figure 9: Normalized critical spin rate vs. the strength of the core of the aggregates for $R_{b}=0.5$ (blue) and $R_{b}=0.7$ (red).

An analysis of the shape of the deformation of the $R_{b}=0.5$ set of aggregates shows that only when the core is at its weakest strength $\left(\sigma_{c c} \leq 0.03\right)$, will the shell present a dent. However, only for $\sigma_{c c}=0.01$, the weakest core we simulated, would the aggregate develop a shape similar to the Itokawa asteroid. Furthermore, the inclusion of a core influenced how the aggregate would deform elastically as a whole. A weak core would allow for a higher elastic deformation, reaching a peak for the homogeneous aggregate $\left(\sigma_{c}=0.1\right)$, to then decrease as the core became stronger (see Table 2). This, however, was contrasted by the much higher ellipticity of these latter aggregates once 
plastic deformation had taken place. This is understandable as aggregates with strong cores would tend to reach higher critical spin rates and ultimately even some granular flow on the surface.

Table 2: Ellipticity of the aggregates at their critical spin rate and before disruption. The homogeneous case is written in bold.

\begin{tabular}{ccc}
\hline$\sigma_{c c}$ & $R_{b}=0.5$ & $R_{b}=0.7$ \\
\hline 0.01 & 0.027 & 0.024 \\
0.03 & 0.026 & 0.024 \\
0.05 & 0.028 & 0.028 \\
0.07 & 0.028 & 0.025 \\
0.09 & 0.029 & 0.027 \\
$\mathbf{0 . 1}$ & $\mathbf{0 . 0 3 3}$ & $\mathbf{0 . 0 3 3}$ \\
0.15 & 0.023 & 0.027 \\
0.2 & 0.022 & 0.023 \\
0.3 & 0.020 & 0.015 \\
0.4 & 0.017 & 0.010 \\
0.5 & 0.015 & 0.009 \\
\hline & &
\end{tabular}

Figure 10 shows the change in the final shape of the aggregates (top: $R_{b}=0.5$ and bottom: $R_{b}=0.7$ ) for $\sigma_{c c}=$ $0.03,0.20$ and 0.40 respectively, from left to right. These images represent cross-sectional cuts perpendicular to the direction of the greatest flow of particles and parallel to the axis of rotation (not actual particle size). They show a side-by-side comparison of the evolution of the shapes of these aggregates after the fission processes have taken place. From them, it is evident that the greater strength of the core facilitates the evolution towards a shape similar to that of asteroid $1999 \mathrm{KW} 4$. However, only a big enough core, namely $R_{b}=0.7$ will be large enough to avoid excessive particle flow towards the equator. So, if the core is not strong enough, it will deform and the final shape will be very round. On the other hand, if it is not large enough, there will be so much particle flow that the final shape will be very flat. Compare with the figures presented by Sánchez (2015).

As for the disruption processes themselves, all of them ejected coherent pieces of material, but not a single one went through as complete a disruption process as that shown for the high cohesion, homogeneous cases presented by Sánchez and Scheeres (2016). This confirms our previous finding that such a disruption pattern can only be present in aggregates with very high cohesive strength and might be relevant to the disaggregation of small granular asteroids (Scheeres, 2018; Hirabayashi et al., 2014). 


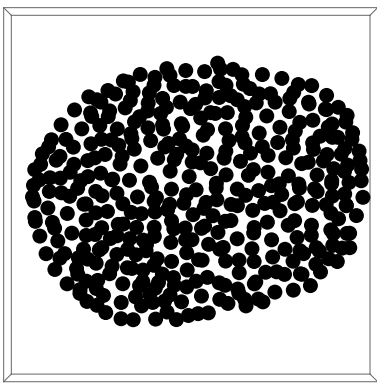

(a) $R_{b}=0.5, \sigma_{c c}=0.03$

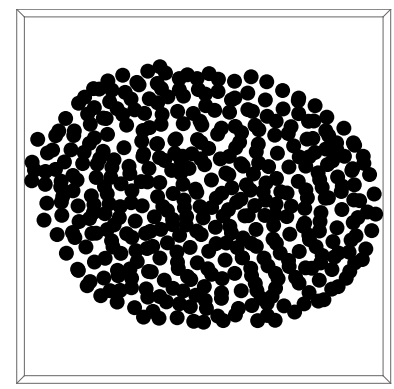

(d) $R_{b}=0.7, \sigma_{c c}=0.03$

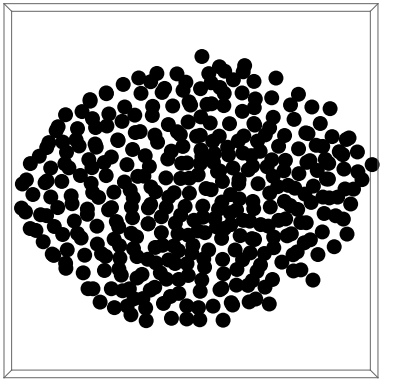

(b) $R_{b}=0.5, \sigma_{c c}=0.20$

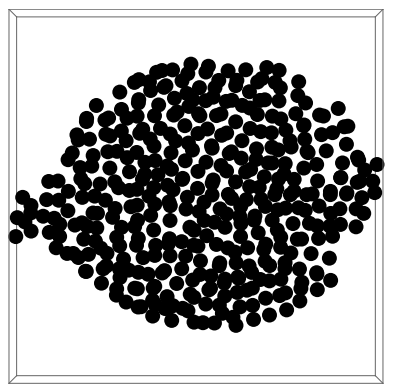

(e) $R_{b}=0.7, \sigma_{c c}=0.20$

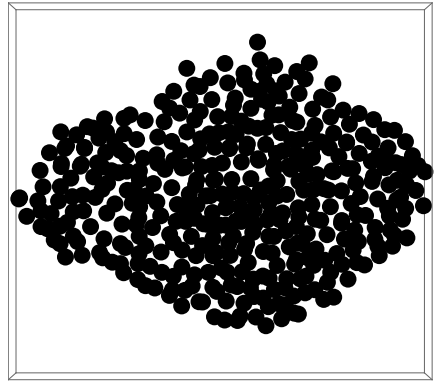

(c) $R_{b}=0.5, \sigma_{c c}=0.40$

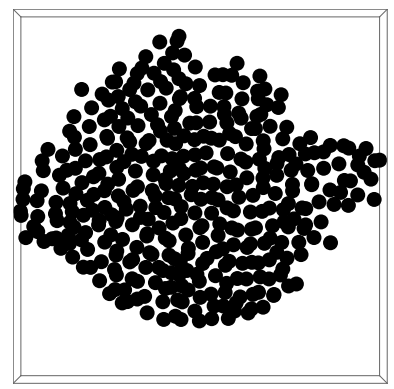

(f) $R_{b}=0.7, \sigma_{c c}=0.40$

Figure 10: Change in the final shape of the aggregates (top: $R_{b}=0.5$ and bottom: $R_{b}=0.7$ ) for $\sigma_{c c}=0.03,0.20$ and 0.40 , from left to right.

\section{Conclusions}

In this paper we have analyzed the effect of the size and relative strength of the core of a self-gravitating aggregate on its rotational evolution.

We have found that for aggregates with weak cores, the size of the core is directly related to the critical spin rate of the aggregates. The larger the core the lower the spin rate had to be to allow for deformation and disruption. This added to the fact that the greatest stress is always located at the centre and a severely weakened core would fail quite easily upon rotation. In no case did any of the weak-core simulations show surface flow to form the equatorial ridge, rather it was formed as the internal core pushed the equatorial region radially outwards. Such a failure pattern will be explicitly searched for in the OSIRIS-REx mission (Scheeres et al., 2016; Tardivel et al., 2018).

Aggregates with weak cores initially generated very symmetrical ellipsoidal shapes that attained some degree of asymmetry after a disruption process had taken place. Noticeably, the aggregate with $R_{b}=0.5$ produced a shape similar to that of asteroid Itokawa; having a dent in the shell that, at greater rotation rates would form the neck of a bi-lobate aggregate. Remarkably, the regions in which the shell stretched the most coincide with the regions in which the asteroid shows an accumulation of smaller regolith and dust. This would mean that, if the Brazil Nut effect took place at some point, this had to happen before Itokawa achieved its present shape. 
To answer the question about the minimum relative strength of the core so that it does not fail before the shell, we took the aggregates with $R_{b}=0.5$ and $R_{b}=0.7$ and varied the strength of their cores. This showed that the core has to be between 3 to 4 times as strong as the shell for this to happen. Additionally, only the weakest of the simulated cores for $R_{b}=0.5$ was able to reproduce the shape of Itokawa even though an aggregate with a $\sigma_{c c}=0.03$ would also produce a dent in its shell.

This work shows that the specific size and relative strength of the core of a heterogeneous aggregate will determine how it will deform and disrupt at elevated spin rates. Different combinations of core size and strength with respect to the aggregate and the shell will produce very different outcomes even if they start from the same configuration. In previous research (Sánchez and Scheeres, 2012, 2014; Hirabayashi et al., 2015; Hirabayashi and Scheeres, 2015; Scheeres, 2015; Sánchez, 2015; Sánchez and Scheeres, 2016; Tardivel et al., 2018), these findings have already been related to observed asteroids such as $1999 \mathrm{KW} 4,1950$ DA, P/2013 R3, P/2013 P5, Geographos, 2000 DP107 and 2008 EV5 as well as to the elevated spin rates of small asteroids and the formation of binary asteroids and asteroid pairs. This implies that the specific shapes, spin rates and surface features that have been observed in asteroids are an expression of their hidden interiors and, as such, should provide insight about the formation and evolution processes of these bodies.

\section{Acknowledgements}

PS would like to thank Kevin Walsh for asking how strong a core should be to be considered strong during the ACM2017 meeting in Uruguay; it was his question that prompted the section about the strength of the core. P.S. and D.J.S. acknowledge support from NASA grant NNX14AL16G from the Near-Earth Object Observation Program and from grants from NASA's SSERVI Institute.

\section{References}

Ai, J., Chen, J.-F., Rotter, J. M., Ooi, J. Y., 2011. Assessment of rolling resistance models in discrete element simulations. Powder Technology $206(3), 269-282$.

URL http://www.sciencedirect.com/science/article/pii/S0032591010005164

Asphaug, E., King, P. J., Swift, M. R., Merrifield, M. R., Mar. 2001. Brazil Nuts on Eros: Size-Sorting of Asteroid Regolith. In: Lunar and Planetary Institute Science Conference Abstracts. Vol. 32 of Lunar and Planetary Inst. Technical Report. pp. 1708-+.

Bottke, Jr., W. F., Richardson, D. C., Michel, P., Love, S. G., 1999. 1620 geographos and 433 eros: Shaped by planetary tides? The Astronomical Journal 117 (4), 1921.

URL http://stacks. iop.org/1538-3881/117/i=4/a=1921

Busch, M. W., Ostro, S. J., Benner, L. A. M., Brozovic, M., Giorgini, J. D., Jao, J. S., Scheeres, D. J., Magri, C., Nolan, M. C., Howell, E. S., Taylor, P. A., Margot, J.-L., Brisken, W., Apr. 2011. Radar observations and the shape of near-Earth ASTEROID 2008 EV5. Icarus 212, 649-660.

Cundall, P., 1971. A computer model for simulating progressive large scale movements in blocky rock systems. In: Proceedings of the International Symposium on Rock Mechanics. Vol. 1. -, Nancy, pp. 129-136. 
Cundall, P. A., Hart, R. D., 1992. Numerical modelling of discontinua. Engineering Computations 9 (2), 101-113.

URL http: //www.emeraldinsight.com/10.1108/eb023851

Fujiwara, A., Kawaguchi, J., Yeomans, D. K., Abe, M., Mukai, T., Okada, T., Saito, J., Yano, H., Yoshikawa, M., Scheeres, D. J., Barnouin-Jha, O., Cheng, A. F., Demura, H., Gaskell, R. W., Hirata, N., Ikeda, H., Kominato, T., Miyamoto, H., Nakamura, A. M., Nakamura, R., Sasaki, S., Uesugi, K., Jun. 2006. The Rubble-Pile Asteroid Itokawa as Observed by Hayabusa. Science 312, 1330-1334.

Herrmann, H., Luding, S., 1998. Modeling granular media on the computer. Continuum Mechanics and Thermodynamics 10, 189-231, $10.1007 / \mathrm{s} 001610050089$.

URL http://dx.doi.org/10.1007/s001610050089

Hirabayashi, M., Sánchez, D. P., Scheeres, D. J., 2015. Internal structure of asteroids having surface shedding due to rotational instability. The Astrophysical Journal 808 (1), 63.

URL http://stacks.iop.org/0004-637X/808/i=1/a=63

Hirabayashi, M., Scheeres, D. J., 2015. Stress and failure analysis of rapidly rotating asteroid (29075) 1950 DA. The Astrophysical Journal Letters 798 (1), L8.

URL http://stacks.iop.org/2041-8205/798/i=1/a=L8

Hirabayashi, M., Scheeres, D. J., Sánchez, D. P., Gabriel, T., 2014. Constraints on the physical properties of main belt comet P/2013 R3 from its breakup event. The Astrophysical Journal Letters 789 (1), L12.

URL http://stacks.iop.org/2041-8205/789/i=1/a=L12

Jewitt, D., Agarwal, J., Li, J., Weaver, H., Mutchler, M., Larson, S., 2014. Disintegrating asteroid P/2013 R3. The Astrophysical Journal Letters 784 (1), L8

URL http: //stacks. iop.org/2041-8205/784/i=1/a=L8

Jewitt, D., Agarwal, J., Weaver, H., Mutchler, M., Larson, S., 2013. The extraordinary multi-tailed main-belt comet P/2013 P5. The Astrophysical Journal Letters 778 (1), L21.

URL http://stacks.iop.org/2041-8205/778/i=1/a=L21

Lowry, S. C., Weissman, P. R., Duddy, S. R., Rozitis, B., Fitzsimmons, A., Green, S. F., Hicks, M. D., Snodgrass, C., Wolters, S. D., Chesley, S. R., Pittichová, J., van Oers, P., Feb. 2014. The internal structure of asteroid (25143) Itokawa as revealed by detection of YORP spin-up. Astronomy and Astrophysics 562, A48.

Miyamoto, H., Yano, H., Scheeres, D. J., Abe, S., Barnouin-Jha, O., Cheng, A. F., Demura, H., Gaskell, R. W., Hirata, N., Ishiguro, M., Michikami, T., Nakamura, A. M., Nakamura, R., Saito, J., Sasaki, S., May 2007. Regolith Migration and Sorting on Asteroid Itokawa. Science 316, 1011-. Naidu, S. P., Margot, J. L., Taylor, P. A., Nolan, M. C., Busch, M. W., Benner, L. A. M., Brozovic, M., Giorgini, J. D., Jao, J. S., Magri, C., 2015. Radar imaging and characterization of the binary near-earth asteroid (185851) 2000 dp107. The Astronomical Journal 150 (2), 54 URL http : //stacks . iop.org/1538-3881/150/i=2/a=54

Perera, V., Jackson, A. P., Asphaug, E., Ballouz, R.-L., 2016. The spherical brazil nut effect and its significance to asteroids. Icarus 278,194 - 203. URL http://www.sciencedirect.com/science/article/pii/S0019103516302986

Rozitis, B., MacLennan, E., Emery, J. P., 08 2014. Cohesive forces prevent the rotational breakup of rubble-pile asteroid (29075) 1950 DA. Nature 512 (7513), 174-176.

URL http://dx.doi.org/10.1038/nature13632

Rubincam, D. P., 2000. Radiative spin-up and spin-down of small asteroids. Icarus 148 (1), 2 - 11.

URL http: //www.sciencedirect.com/science/article/B6WGF-45FCNK6-2/2/17460f69fc41f03668a0024ad24bb8d5

Sánchez, D. P., Scheeres, D. J., 2012. Dem simulation of rotation-induced reshaping and disruption of rubble-pile asteroids. Icarus 218 (2), 876 894.

URL http://www.sciencedirect.com/science/article/pii/S0019103512000292 
Sánchez, P., 008 2015. Asteroid evolution: Role of geotechnical properties. Proceedings of the International Astronomical Union 10 (S318), $111-121$.

URL https://www.cambridge.org/core/article/asteroid-evolution-role-of-geotechnical-properties/ CEFD7D6A46A7E74A06F90800DC0E1F6F

Sánchez, P., Scheeres, D. J., 2011. Simulating asteroid rubble piles with a self-gravitating soft-sphere distinct element method model. The Astrophysical Journal 727 (2), 120.

URL http://stacks. iop.org/0004-637X/727/i=2/a=120

Sánchez, P., Scheeres, D. J., 2014. The strength of regolith and rubble pile asteroids. Meteoritics \& Planetary Science 49 (5), $788-811$.

URL http://dx.doi.org/10.1111/maps.12293

Sánchez, P., Scheeres, D. J., 2016. Disruption patterns of rotating self-gravitating aggregates: A survey on angle of friction and tensile strength. Icarus $271,453-471$.

URL http://www.sciencedirect.com/science/article/pii/S0019103516000208

Sánchez, P., Scheeres, D. J., Swift, M. R., Mar. 2010. Impact driven size sorting in self-gravitating granular aggregates. In: Lunar and Planetary Institute Science Conference Abstracts. Vol. 41 of Lunar and Planetary Inst. Technical Report. pp. 2634-+.

Scheeres, D., 2015. Landslides and mass shedding on spinning spheroidal asteroids. Icarus 247 (0), 1 - 17.

URL http://www.sciencedirect.com/science/article/pii/S0019103514004795

Scheeres, D., 2018. Disaggregation of small, cohesive rubble pile asteroids due to YORP. Icarus 304, 183 - 191, asteroids and Space Debris.

URL http://www.sciencedirect.com/science/article/pii/S0019103517300404

Scheeres, D., Gaskell, R., 2008. Effect of density inhomogeneity on yorp: The case of itokawa. Icarus 198 (1), 125 - 129.

URL http://www.sciencedirect.com/science/article/pii/S0019103508002790

Scheeres, D., Hesar, S., Tardivel, S., Hirabayashi, M., Farnocchia, D., McMahon, J., Chesley, S., Barnouin, O., Binzel, R., Bottke, W., Daly, M., Emery, J., Hergenrother, C., Lauretta, D., Marshall, J., Michel, P., Nolan, M., Walsh, K., 2016. The geophysical environment of bennu. Icarus 276 (Supplement C), $116-140$.

URL http://www.sciencedirect.com/science/article/pii/S0019103516300598

Scheeres, D. J., Aug. 2007. Rotational fission of contact binary asteroids. Icarus 189, 370-385.

Silbert, L. E., Ertaş, D., Grest, G. S., Halsey, T. C., Levine, D., Plimpton, S. J., Oct 2001. Granular flow down an inclined plane: Bagnold scaling and rheology. Phys. Rev. E 64 (5), 051302.

Tardivel, S., Sánchez, P., Scheeres, D. J., 2018. Equatorial cavities on asteroids, an evidence of fission events. Icarus 304, 192 - 208, asteroids and Space Debris.

URL http://www.sciencedirect.com/science/article/pii/S0019103517300106

Yano, H., Kubota, T., Miyamoto, H., Okada, T., Scheeres, D., Takagi, Y., Yoshida, K., Abe, M., Abe, S., Barnouin-Jha, O., Fujiwara, A., Hasegawa, S., Hashimoto, T., Ishiguro, M., Kato, M., Kawaguchi, J., Mukai, T., Saito, J., Sasaki, S., Yoshikawa, M., Jun. 2006. Touchdown of the Hayabusa Spacecraft at the Muses Sea on Itokawa. Science 312, 1350-1353. 